\title{
Robust implementations of quantum repeaters
}

\author{
Alexander Klein, Uwe Dorner, Carolina Moura Alves, and Dieter Jaksch \\ Clarendon Laboratory, University of Oxford, Parks Road, Oxford OX1 3PU, United Kingdom
}

(Received 8 November 2005; published 23 January 2006)

\begin{abstract}
We show how to efficiently exploit decoherence free subspaces (DFSs), which are immune to collective noise, for realizing quantum repeaters with long-lived quantum memories. Our setup consists of an assembly of simple modules and we show how to implement them in systems of cold, neutral atoms in arrays of dipole traps. We develop methods for realizing robust gate operations on qubits encoded in a DFS using collisional interactions between the atoms. We also give a detailed analysis of the performance and stability of all required gate operations and emphasize that all modules can be realized with current or near future experimental technology.
\end{abstract}

DOI: 10.1103/PhysRevA.73.012332

PACS number(s): 03.67.Mn, 03.67.Hk, 03.67.Pp, 32.80.Pj

\section{INTRODUCTION}

One of the major obstacles in the realization of quantum information processors (QIP) is decoherence, caused by the unwanted interaction of the system with its environment. Even the feasibility of simple special purpose quantum devices, such as quantum repeaters, is significantly affected by the presence of decoherence and a special precaution to counteract the resulting loss of quantum information has to be taken. A number of concepts have been developed to fight decoherence and increase the reliability of QIP including active error correction [1-3] and fault tolerant quantum computing [4]. Aside from this it was shown that, dependent on the type of interaction processes between the qubits and their environment, subspaces of the system's Hilbert space can exist which are immune to decoherence processes induced by this interaction [5-8]. These decoherence free subspaces are a method of passive error correction or error prevention and can significantly increase the lifetime of quantum information and reliability of QIP as already demonstrated with ion traps, NMR and optical methods [9-16]. However, this robustness makes states within the decoherence free subspaces (DFS) difficult to manipulate via controlled operations and, in general, complicated interactions with a DFS are necessary for realizing universal QIP [8].

A particularly promising application of DFSs are quantum repeaters $[17,18]$ which require very long quantum information storage times but only a limited set of quantum manipulations. In this paper we show that the quantum operations necessary for a quantum repeater can be realized on DFS qubits with nearest neighbor hopping $[19,20]$ and interactions with ancilla qubits [21] only, thus circumventing the difficulties usually associated with the usage of DFS [8]. Quantum repeaters are used to distribute maximally entangled pairs of qubits over long distances which is a necessary prerequisite for important applications such as quantum cryptography [22] and quantum teleportation [23]. They are thus considered one of the most important near future special purpose QIP.

The basic idea of a quantum repeater is to divide the transmission line into segments with a length of the order of the attenuation length of the channel. On each segment entangled particle pairs are created and by applying entangle- ment swapping [24] and purification protocols $[17,25,26]$ entangled pairs of larger distances are produced. By repeating these steps a distant pair of qubits with high entanglement fidelity is obtained. Entanglement purification, distillation, and concentration have been realized in a number of seminal experiments [27-30] using photonic states for encoding the qubits. Techniques for converting flying photonic qubits into atomic ones via cavity-enhanced interactions $[31,32]$ or the usage of atomic ensembles $[33,34]$ have been proposed. Very recently a demonstration of long distance entanglement of massive particles has been achieved [35].

Here we develop a proposal for a quantum repeater with DFS quantum memory using the entanglement purification scheme introduced in $[17,36]$ (also known as the "Innsbruck protocol"). During the purification procedure the entanglement of a primary pair of qubits is increased by sacrificing auxiliary entangled qubit pairs. The entanglement fidelity of the final entangled qubit pair does not converge to 1 , but to a value which depends on the fidelity of the initial primary pair and on the constant fidelity of the auxiliary pairs. However, the scheme $[17,36]$ is favorable from a pragmatic point of view since it requires fewer physical resources than the purification schemes used in [25,26,37]. On the other side, the protocol $[17,36]$ takes longer and thus quantum information has to be stored reliably for the whole duration of the protocol. We overcome this apparent disadvantage by the usage of DFS qubits of four neutral atoms which form the key elements of our DFS quantum repeater.

Our scheme is based on current and near future technology and is motivated by the extraordinary progress of the field of cold, neutral atoms in dipole traps over the past few years both on the theoretical [38-41] and on the experimental $[42,43]$ side. Atomic quantum registers have been realized in arrays of microlenses $[44,45]$ and by exploiting the superfluid to Mott-Insulator transition in optical lattices $[38,46,47]$, single and many atoms have been manipulated coherently $[48,49]$, atoms stored in standing waves have been transported over macroscopic distances using conveyor belt techniques [50-53], and multipartite entangled states of atoms were created $[49,54]$. Furthermore, extremely versatile technologies such as optical tweezers and holographic traps which are controlled by liquid crystal spatial light modula- 


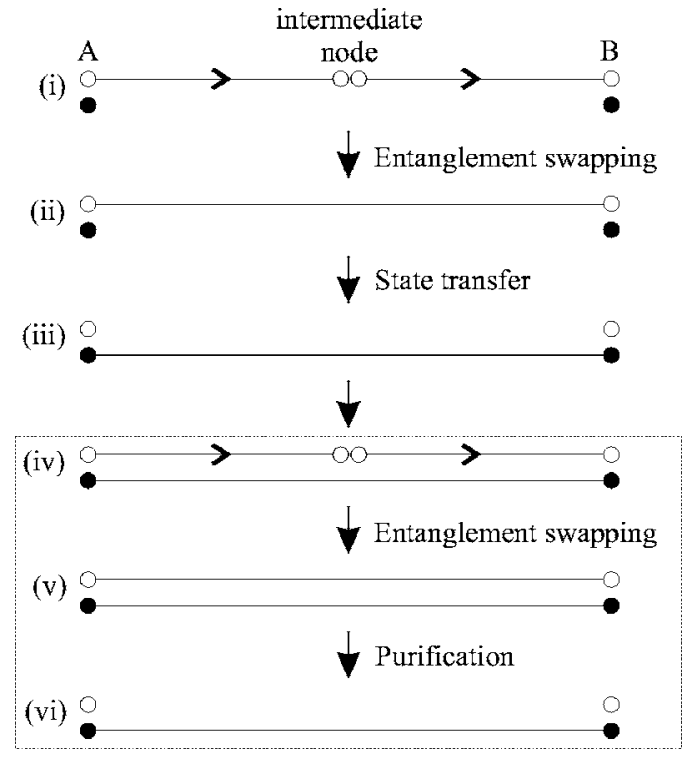

FIG. 1. Illustration of the entanglement purification scheme. Pairs of entangled flying qubits (open circles) are created via entanglement swapping. These pairs are then used to purify an entangled pair of DFS qubits (solid circles; see text). The framed steps (iv)-(vi) are repeated until a desired entanglement fidelity is achieved.

tors [55-57] are currently developed. They allow for essentially arbitrary designs of optical potentials.

We will show that a DFS quantum repeater can be assembled from modules which only require rotations of the logical DFS qubits around the $x$ and $z$ axes by angles $\pi$ and $\pi / 2$ and a controlled phase (CPHASE) gate where an ancilla atom acts as the control qubit. The single qubit rotations will be realized by selectively lowering potential barriers between atoms and the system will not leave the DFS during these gates. The ancilla qubit, which is required to interact with a DFS qubit for realizing the CPHASE gate, will not be decoherence free. However, we will show that its influence on the DFS memory is sufficiently small to allow for gate fidelities better than $98.7 \%$. Furthermore, we will show that our scheme allows for deviations of the system parameters of a few percent without significantly affecting the gate fidelities.

This paper is structured as follows: In Sec. II we will briefly summarize the repeater protocol and show how DFSs can be embedded into this scheme. The goal of this section is to show what resources are needed for the implementation of the quantum repeater. In Sec. III we propose methods of how the operations of Sec. II can be physically realized with cold, neutral atoms in arrays of dipole traps. The results are summarized in Sec. IV.

\section{QUANTUM REPEATERS WITH DECOHERENCE FREE SUBSPACES}

In the following we will describe the purification and repeater protocol of Refs. $[17,36]$ and extend it by using DFS. The basic steps of the purification scheme are illustrated in Fig. 1. Only one quantum channel (represented by the lines

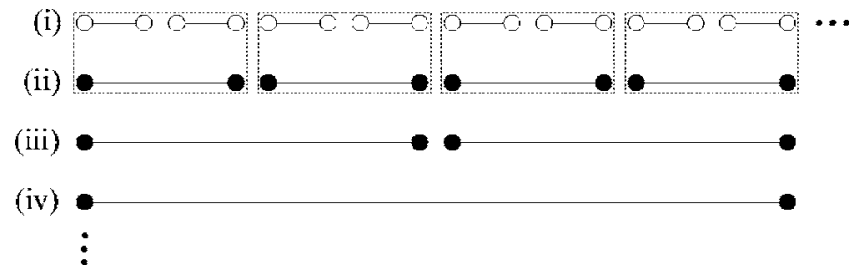

FIG. 2. The quantum repeater scheme. The dashed boxes indicate the subroutine shown in Fig. 1. The created entangled DFS qubit pairs are used to purify further DFS qubit pairs [in line (iii)]. The resulting pairs are then used to purify the pairs of line (iv), and so on.

with an arrow) and two DFS qubits (represented by the solid circles) are needed. The goal is to share a high fidelity Bell state between $A$ and $B$ which is done by transmission of "one-half" of a maximally entangled flying qubit pair [open circles in Fig. 1(i)]. In general, the flying qubit (e.g., a photon) is not directly sent from $A$ to $B$ since the present available transmission quantum channels (particularly optical fibers) degrade the entanglement of the flying qubit pair exponentially with the distance between the nodes. Thus, if the channel is too long, the minimum fidelity required for the successful application of the purification protocol $\left(F_{\min }\right)$ is not achieved. To overcome this problem a sufficiently high number of intermediate nodes between $A$ and $B$ is introduced such that the distance between two nodes is of the order of the optical fiber's attenuation length [36]. Entangled flying qubit pairs are prepared at each of the intermediate nodes and transmitted to the next node. In Fig. 1 this is illustrated for the example of one intermediate node. After this, entanglement swapping [24] at all intermediate nodes is performed which yields a partially entangled pair of flying qubits between $A$ and $B$ and its state is transferred to a DFS qubit pair [step (ii)]. Finally, as in (i) and (ii), auxiliary entangled qubit pairs are generated which are sacrificed in order to increase the entanglement of the DFS qubit pair [step (iv)-(vi)]. Steps (iv)-(vi) are repeated until a desired degree of entanglement is obtained for the DFS qubit pair.

However, a straightforward extension of this scheme in order to connect arbitrary separated points $A$ and $B$ is not possible since the distance between the intermediate nodes has to be chosen such that the fidelity of the final pair is not lower than $F_{\min }$. This is, in general, not possible if the distance between $A$ and $B$ is too large. Therefore, a quantum repeater concept by means of a nested purification scheme was proposed $[17,36]$ which uses the above purification protocol to create a number of entangled DFS qubit pairs which are then used to purify a lower number of additional DFS pairs with greater distance. These pairs are then again used to purify an even lower number of pairs, etc. until merely one entangled pair is left. This procedure is illustrated in Fig. 2: The boxes stretching over lines (i) and (ii) represent the purification protocol of Fig. 1. The entangled pairs of line (ii) are then used to purify the pairs in line (iii) in the same way as in Fig. 1 except that the flying qubits have to be replaced by DFS qubits, i.e., entanglement swapping between DFS qubits, a state transfer between DFS qubits [to initialize the pairs in line (iii) and (iv)], and purification by using en- 
tangled DFS pairs have to be performed. The diagram shown in Fig. 2 can be extended in horizontal and vertical directions such that an arbitrary distance between the final pair can be achieved. It was shown that the number of (DFS) qubits necessary for the scheme increases merely logarithmically with the distance of the final entangled pair $[17,36]$.

In summary, the purification procedure shown in Fig. 1 can be realized by assembling the following modules: (0) The creation of pairs of flying qubits and performing entanglement swapping between them, (1) the realization of two DFS qubits, (2) the transfer of the state of the flying qubits to the DFS qubits, and (3) the actual purification of the entangled DFS qubit pair. Additionally, for the nested purification protocol, i.e., for the full implementation of the quantum repeater, we have to (4) perform entanglement swapping between neighboring DFS qubits, (5) we need a state transfer between DFS qubits [analogously to module (2)], and (6) we must perform entanglement purification using two DFS qubit pairs. Module (0) can be implemented using standard quantum optical methods as demonstrated in [58-62]. It does not involve DFS qubits and will thus not be discussed further in this paper.

In the following we will show how each of the remaining modules can be realized. For the operations using ancilla qubits and thus leaving the DFS we then calculate the achievable fidelities.

\section{A. Realization of the repeater modules}

\section{Module 1: Decoherence free memory and single qubit rotations}

The main sources of decoherence in systems of cold atoms are fluctuations of the optical potential and external electromagnetic (stray-)fields [63]. These sources are homogeneous across the region of the physical qubits and the interaction with them thus only depends on their internal states. The Hamiltonian describing the situation is given by

$$
H_{I}=\sum_{i} \sigma_{i}^{x} B_{x}+\sigma_{i}^{y} B_{y}+\sigma_{i}^{z} B_{z} .
$$

Here, $i$ labels the physical qubits, $\mathbf{B}=\left(B_{x}, B_{y}, B_{z}\right)$ describes the fields and fluctuations, and the $\sigma_{i}$ are Pauli matrices acting on the $i$ th physical qubit.

The corresponding decoherence free subspace is spanned by the states with total zero angular momentum, and the lowest number of physical qubits giving a twofold degenerate DFS is four. The DFS qubit is then represented by $[8,64]$

$$
\begin{gathered}
|0\rangle_{L}=\frac{1}{2}(|01\rangle-|10\rangle) \otimes(|01\rangle-|10\rangle), \\
|1\rangle_{L}=\frac{1}{2 \sqrt{3}}\left[2|1100\rangle+2|0011\rangle-(|01\rangle+|10\rangle)^{\otimes 2}\right],
\end{gathered}
$$

where $|i j k l\rangle=|i\rangle_{1}|j\rangle_{2}|k\rangle_{3}|l\rangle_{4}$ with $i, j, k, l=0,1$ describes the computational basis states of the four physical qubits. Each of the four physical qubits is represented by two internal states of an atom which are trapped in an array of dipole traps, for example, an optical lattice. By the method described in Sec. III A the system is prepared in the logical

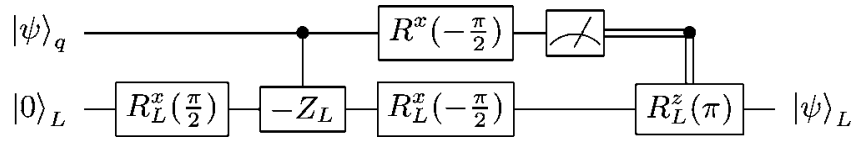

FIG. 3. Quantum circuit for the state transfer of the auxiliary qubit to the DFS qubit.

state $|0\rangle_{L}$ and in Sec. III B it is shown that by tuning the system parameters appropriately we can implement the Hamiltonians $H_{X}$ and $H_{Z}$ with

$$
\begin{gathered}
H_{X}=X_{L}=\frac{1}{\sqrt{3}}\left(V_{12}+2 V_{23}\right), \\
H_{Z}=Z_{L}=-V_{12},
\end{gathered}
$$

where $X_{L}\left(Z_{L}\right)$ is the $X$ gate ( $Z$ gate) for the logical qubit and $V_{i j}$ are permutations of the physical qubits $i$ and $j$. Note that the logical $Z$ gate can also be represented as $Z_{L}=-V_{34}$. Since the DFS defined by Eq. (2) is invariant under permutations of the physical qubits [8], the time evolution of the system generates rotations

$$
\begin{aligned}
R_{L}^{x}(\theta) & =e^{-\mathrm{i} \theta X_{L} / 2}, \\
R_{L}^{z}(\theta) & =e^{-\mathrm{i} \theta Z_{L} / 2}
\end{aligned}
$$

without ever leaving the DFS, where $\theta=2 t$ and $t$ is the time necessary for performing the rotations. The index $L$ indicates that these are operations on the logical qubit (in the following we will also use this notation for operations on physical qubits, where we omit the index $L$ ).

Although it is not required in the repeater protocol, we can thus perform arbitrary rotations of the logical qubit (and hence we can prepare arbitrary states in the DFS) since every two level unitary operator can be decomposed in $R_{L}^{x}$ and $R_{L}^{z}$ [65].

\section{Module 2: State transfer between flying and DFS qubit}

The quantum circuit for the state transfer of the flying qubit to the logical qubit is shown in Fig. 3. The state of the flying qubit is denoted as $|\psi\rangle_{q}=\alpha|0\rangle_{q}+\beta|1\rangle_{q}$. As described in Sec. III the state of the flying qubit (a photon) is first transferred to an appropriate internal state of an auxiliary atom. The state of this atom then controls the interaction between physical qubits 3 and 4 leading to a controlled SWAP operation between these atoms. Since $Z_{L}=-V_{34}$, a controlled SWAP operation of the physical qubits 3 and 4 leads to a controlled $\left(-Z_{L}\right)$ (CPHASE) gate on the logical qubit. As can be seen from Fig. 3, apart from rotations given in Eqs. (5) and (6), we need to perform a rotation $R^{x}(-\pi / 2)$ and a measurement of the control atom which can be done by standard techniques such as laser induced Rabi oscillations and state selective laser excitations. Eventually, the state of the atom is transferred to the logical qubit, i.e., $|\psi\rangle_{L}=\alpha|0\rangle_{L}+\beta|1\rangle_{L}$.

\section{Module 3: Entanglement purification}

The local operations required for the entanglement purification are shown in Fig. 4. 


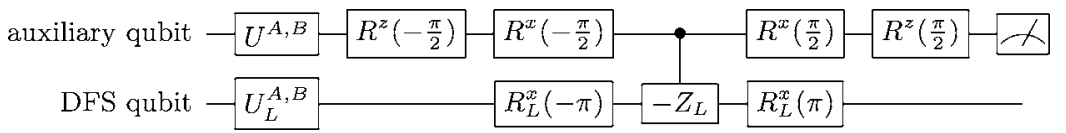

FIG. 4. Quantum circuit for entanglement purification. The procedure is performed on both sides, $A$ and $B$.
This circuit corresponds to the scheme proposed in [26], but here it is expressed in terms of operations that we can implement in our setup. The circuit has to be applied on both ends of the transmission line $A$ and $B$, where

$$
U_{L}^{A}=\left(U_{L}^{B}\right)^{\dagger}=R_{L}^{x}(\pi / 2)
$$

As in Sec. II A 2, the auxiliary qubit is again an atom that controls a phase operation on the logical qubit. If the outcome of the measurements at $A$ and $B$ coincide we end up with an entangled DFS qubit pair of higher fidelity. If they do not coincide the purification procedure has to be repeated.

\section{Module 4: Entanglement swapping between DFS qubits}

Entanglement swapping between neighboring DFS qubit pairs can be done by a Bell measurement and subsequent local operations $R_{L}^{x}(\pi / 2)$ and $R_{L}^{z}(\pi / 2)$ that are controlled by the outcome of the measurement [36]. The Bell measurement can be decomposed in a controlled not (CNOT) gate, a Hadamard gate, and a measurement in the computational basis [the Hadamard gate can be replaced by, e.g., the sequence $\left.R_{L}^{z}(\pi / 2) R_{L}^{x}(\pi / 2) R_{L}^{z}(\pi / 2)\right]$.

The implementation of a decoherence free CNOT operation is relatively complicated including lengthy sequences of twoparticle interactions with pairwise individual tuning [8]. Therefore we use an ancilla atom (as in Secs. II A 2 and II A 3) to mediate the interaction between different DFS qubits. A possible quantum circuit for a CNOT gate between DFS qubits is shown in Fig. 5. The centerline in the circuit represents the ancilla qubit. The shown circuit uses only gates that we can implement in our scheme and leads to a CNOT gate with the upper DFS qubit as the control qubit. We will discuss in Sec. II B how the decoherence of the auxiliary atom influences the fidelity of this operation.

For the measurement of the DFS qubit we use the same ancilla as for the CNOT gate. The corresponding circuit is shown in Fig. 6. The final measurement of the ancilla is equivalent to a measurement of the DFS qubit.

\section{Modules 5 and 6: State transfer between DFS qubits and entanglement purification using pairs of DFS qubits}

The state transfer of one DFS qubit to another can be done analogously to Fig. 3, where $|\psi\rangle_{q}$ has to be replaced by $|\psi\rangle_{L}$. The necessary CPHASE gate between DFS qubits can be constructed similarly as the CNOT gate: We merely have to omit the single qubit rotations in the last line of Fig. 5. Also the entanglement purification scheme of Fig. 4 can be adapted in this way. Again, the auxiliary qubit is replaced by a DFS qubit and the CPHASE (or CNOT) gate is realized as described above.

\section{B. Operation time and decoherence}

Some of the above operations require the use of a single auxiliary qubit which is not decoherence free. Entangling this qubit with the DFS qubits will therefore lower the overall fidelity of the corresponding gate operations on the DFS qubits. The auxiliary qubit is represented in our scheme by two long-lived internal states of an atom and thus the major experimentally expected decoherence mechanism is dephasing [66]. This process is described by the master equation

$$
\partial_{t} \varrho_{\mathrm{aux}}=-\frac{i}{\hbar}\left[\hat{H}, \varrho_{\mathrm{aux}}\right]+\frac{\gamma}{2}\left(\sigma_{z} \varrho_{\mathrm{aux}} \sigma_{z}-\varrho_{\mathrm{aux}}\right),
$$

where $\varrho_{\text {aux }}$ is the density matrix of the auxiliary atom, $\hat{H}$ is the Hamiltonian of the system, $1 / \gamma$ characterizes the decoherence time, and $\sigma_{z}$ is a Pauli matrix. Decoherence of the DFS qubits is not taken into account here.

A quantitative measure of the operation fidelity is derived from Ref. [65]. A distance measure of two density matrices $\varrho, \sigma$ is given by

$$
f(\varrho, \sigma)=(\operatorname{Tr} \sqrt{\sqrt{\sigma} \varrho \sqrt{\sigma}})^{2},
$$

where, in contrast to [65], we use the squared trace since for pure states this corresponds to a probability, i.e., the absolute square of the overlap of the two states. The operation-(or gate-) fidelity can be derived from this quantity: Let $\mathcal{E}$ be a quantum operation describing a quantum circuit where decoherence is taken into account, and $\mathcal{E}_{0}$ the same operation without decoherence. We define the operation fidelity by

$$
F\left(\mathcal{E}_{0}, \mathcal{E}\right)=\min _{\varrho} f\left(\mathcal{E}_{0}(\varrho), \mathcal{E}(\varrho)\right)
$$

where it is sufficient to minimize over pure input states $\varrho$ [65].

Let us first consider the state transfer from the atom to the DFS qubit (Fig. 3). To minimize decoherence effects, the $R_{L}^{x}(\pi / 2)$ and $R_{L}^{x}(-\pi / 2)$ gates are applied before the auxiliary atom interacts with the DFS qubit and after the measurement, respectively. In addition, we neglect decoherence during the $R^{x}(-\pi / 2)$ rotation since the time to perform such a gate on one atom is, in general, much smaller than the expected decoherence time $1 / \gamma$ (see Sec. III D). Thus, decoherence occurs mainly during the CPHASE gate. We examined the dynamics of the system during the application of the

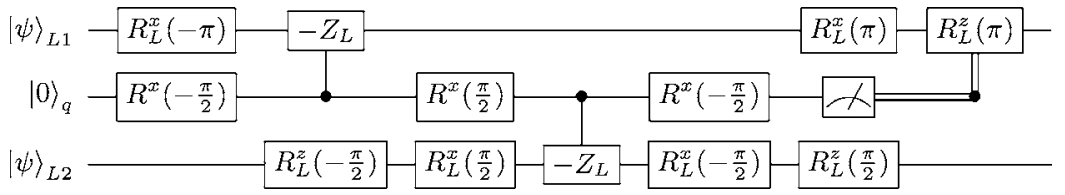

FIG. 5. Quantum circuit for a CNOT operation between two logical qubits. 


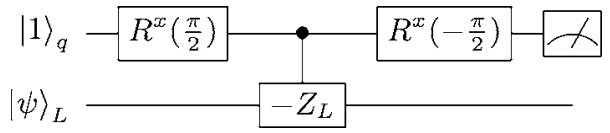

FIG. 6. Measurement of the logical qubit.

CPHASE gate by solving Eq. (8) analytically. The resulting gate fidelity for the CPHASE operation is given by

$$
F_{\text {CPHASE }}\left(\mathcal{E}_{0}, \mathcal{E}\right)=\frac{1+e^{-\gamma t}}{2} \geqslant 1-\frac{\gamma t}{2} .
$$

As will be discussed in Sec. III D this behavior is sufficient to ensure a working repeater scheme.

Note that, since unitary operations do not change the fidelity of the circuits [65], all operations which only involve one CPHASE gate have the same fidelity given in Eq. (11). This includes particularly the operations shown in Figs. 4 and 6 .

In a similar way we calculated the fidelity of the CNOT gate between the two DFS qubits (Fig. 5). Again, all $R_{L}^{(x, z)}$ gates are applied before the auxiliary atom interacts with the DFS qubit and after the measurement, respectively. During these periods the auxiliary atom is not entangled with either DFS qubit and thus its decoherence has no influence on them. Furthermore, we assume again that decoherence during the $R^{(x, z)}$ rotations can be neglected. The gate fidelity of the CNOT operation is then given by

$$
F_{\mathrm{CNOT}}\left(\mathcal{E}_{0}, \mathcal{E}\right)=\left(\frac{1+e^{-\gamma t}}{2}\right)^{2} \geqslant 1-\gamma t,
$$

where $t$ is the time which is needed to perform one CPHASE gate. Since the two DFS qubits are not entangled before the CNOT gate is applied it is sufficient to use only pure, unentangled states for the minimization [65]. The fidelity for a CPHASE gate between two DFS qubits is the same as for the CNOT gate because they differ only by unitary operations. As in the case of the CPHASE gate, the decay of the CNOT fidelity is slow enough to ensure a working quantum repeater (see Sec. III D).

The fidelity of the state transfer between two DFS qubits described in Sec. II A 5 can also be calculated by explicitly solving the time evolution. The state transfer fidelity $F_{\mathrm{ST}}$ is given by

$$
F_{\mathrm{ST}}\left(\mathcal{E}_{0}, \mathcal{E}\right)=\frac{1}{4}\left(1+e^{-2 \gamma t}\right)\left(1+e^{-\gamma t}\right) \geqslant 1-\frac{3}{2} \gamma t .
$$

The fidelity of the entanglement purification using pairs of DFS qubits (see Sec. II A 5) is calculated using the chaining property for fidelity measures [65]. Assuming that the two DFS qubits on one side $A$ or $B$ are not entangled with each other we find as a lower bound

$$
F_{\mathrm{EP}}\left(\mathcal{E}_{0}, \mathcal{E}\right) \geqslant 1-\left(\frac{3}{2}+\sqrt{2}\right) \gamma t
$$

In both formulas, Eqs. (13) and (14), the time $t$ is again the time needed for performing one CPHASE gate. As we will show in Sec. III D all of these infidelities are small for typical experimental decoherence times.

In summary, we have shown in this section that the DFS quantum repeater can be divided into simple modules which

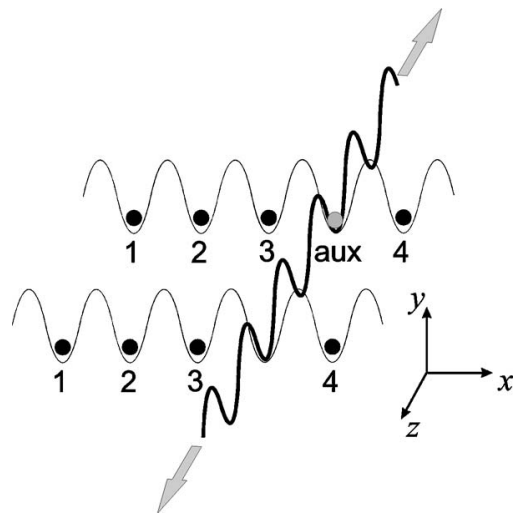

FIG. 7. Schematic picture of a repeater node. The four register atoms per qubit (black circles) are stored in a one-dimensional array of dipole traps, e.g., an optical lattice. Between the third and fourth atom there is a free lattice site in which the auxiliary atom (gray) can be moved, e.g., via an optical conveyor belt.

require (apart from single qubit rotations and measurements of an ancilla atom which can be realized by standard methods) merely the operations $R_{L}^{x}( \pm \pi / 2), R_{L}^{x}( \pm \pi), R_{L}^{z}( \pm \pi / 2)$, $R_{L}^{z}(\pi)$, and a CPHASE gate on a DFS qubit with the ancilla atom as control qubit. In the following section we proceed by describing how these basic operations can be implemented in systems of trapped neutral atoms.

\section{PHYSICAL IMPLEMENTATION IN ARRAYS OF DIPOLE TRAPS}

The physical system we consider for the implementation of a DFS qubit is a one-dimensional chain of four atoms in an array of optical dipole traps, e.g., an optical lattice [38] with one atom per site. Each of the atoms has two long-lived internal states $a$ and $b$ which encode a physical qubit $\hat{a}^{\dagger} \mid$ vac $\rangle \equiv|0\rangle, \hat{b}^{\dagger} \mid$ vac $\rangle \equiv|1\rangle$. In Fig. 7 two DFS qubits are schematically shown. The thick, diagonal line represents an optical conveyor belt [53] storing the auxiliary atom. This atom can be transported to the DFS strings where it occupies an empty site between the third and the fourth atom, which is necessary for the CPHASE operations described in Sec. II. Aside from this, the auxiliary atom is needed for the state transfer of the flying qubit to the DFS qubit: The flying qubits in our repeater scheme are represented by entangled photon pairs. After a photon arrives at a repeater node its state is transferred to the auxiliary atom, e.g., by a cavity-enhanced interaction $[31,32]$. The atom is then moved to the DFS qubit and the operation shown in Fig. 3 is performed. In order to implement the rotations on the DFS qubits we also have to be able to selectively lower lattice barriers between certain lattice sites. This can be done by using suitable superlattices or by applying single laser pulses as described in [19]. The addressability of single atoms is only required for the initialization of the register, cf. Sec. III A.

The general Hamiltonian for a one-dimensional atom chain for $M$ lattice sites (without auxiliary atom) is given by [38] 


$$
\begin{aligned}
\hat{H}= & \sum_{j=1}^{M-1}\left(-J_{j}^{(a)} \hat{a}_{j}^{\dagger} \hat{a}_{j+1}-J_{j}^{(b)} \hat{b}_{j}^{\dagger} \hat{b}_{j+1}+\text { H.c. }\right) \\
& +\sum_{j=1}^{M}\left(\frac{U_{a}}{2} \hat{a}_{j}^{\dagger} \hat{a}_{j}^{\dagger} \hat{a}_{j} \hat{a}_{j}+\frac{U_{b}}{2} \hat{b}_{j}^{\dagger} \hat{b}_{j}^{\dagger} \hat{b}_{j} \hat{b}_{j}+U_{a b} \hat{a}_{j}^{\dagger} \hat{a}_{j} \hat{b}_{j}^{\dagger} \hat{b}_{j}\right),
\end{aligned}
$$

where $J_{j}^{(\alpha)}$ are state dependent hopping matrix elements from site $j$ to site $j+1$ and $U_{a, b}, U_{a b}$ are state dependent on-site interaction strengths. We should mention that for the rotations $R_{L}^{x}$ and $R_{L}^{z}$ and for the CPHASE gate the hopping constants for both kinds of atoms are required to be the same, $J_{j}^{(a)}=J_{j}^{(b)}$, as well as $U_{a}=U_{b}=U_{a b}$, cf. also Appendix A. Apart from the initialization of the register we need $M=5$ lattice sites.

In the following subsections we will show how the register can be prepared in state $|0\rangle_{L}$ and how the rotations $R_{L}^{(x, z)}$ and the CPHASE gate can be implemented. These three ingredients are sufficient to realize the repeater protocol.

\section{A. Initialization of state $|0\rangle_{L}$}

The preparation of state $|0\rangle_{L}$ requires the creation of a pair of singlet states $(|01\rangle-|10\rangle) / \sqrt{2}$. These states are decoupled from global operations but can be realized by local operations and by selectively lowering the potential barriers between neighboring atoms. Starting out from the product state $|0110\rangle$ the potential barrier between the first and second atom and between the third and fourth atom are lowered (e.g., via a superlattice potential). This generates two double well type potentials. The parameters of the Hamiltonian Eq. (15) are then given by

$$
J_{2}=0, \quad J_{1,3}^{(a)}=J_{1,3}^{(b)}=J, \quad U_{a}=U_{b}=U_{a b}=U
$$

Assuming that $J / U$ is sufficiently small (see also Sec. III B), states with two atoms in one site can be adiabatically eliminated leading to an effective Hamiltonian [67] of the form

$$
H_{\mathrm{ini}}=-\frac{J^{2}}{U}\left(\sigma_{1}^{x} \sigma_{2}^{x}+\sigma_{1}^{y} \sigma_{2}^{y}+\sigma_{1}^{z} \sigma_{2}^{z}+\sigma_{3}^{x} \sigma_{4}^{x}+\sigma_{3}^{y} \sigma_{4}^{y}+\sigma_{3}^{z} \sigma_{4}^{z}\right)
$$

Turning this Hamiltonian on for a time $t=\pi \hbar U / 8 J^{2}$ we obtain the state $(|01\rangle+\mathrm{i}|10\rangle) \otimes(|10\rangle+\mathrm{i}|01\rangle) / 2$. Finally, a local operation on the first and fourth physical qubit of the form $\exp \left(-\mathrm{i} \pi \sigma_{1}^{z} / 4\right)$ and $\exp \left(-\mathrm{i} \pi \sigma_{4}^{z} / 4\right)$, respectively, yields the desired product of singlet states. Alternatively, methods proposed in [68] can be used to realize the state $(|01\rangle+|10\rangle)$ $\otimes(|10\rangle+|01\rangle) / 2$. Local single qubit rotations of the first and fourth qubit can be used to bring this state into the desired from. If laser addressing of single physical qubits in adjacent lattice sites is not possible we can move the first and the fourth atom into their neighboring, free sites (and back again) by lowering the corresponding potential barriers.
In order to write information into the register we require a free lattice site between the third and fourth atom. Hence, in a last step the fourth atom is moved into the fifth lattice site by lowering the potential barrier between the fourth and fifth site.

\section{B. Rotations of the logical qubit}

In this section we analytically investigate how to implement the rotations $R_{L}^{x}$ and $R_{L}^{z}$ in the two limiting cases of a very high interaction between the register atoms and a vanishing interaction. The system is simulated numerically to check our results.

\section{Analytical treatment}

The Hamiltonian $H_{Z}$ is, apart from a sign, equivalent to a swap of the first two atoms $V_{12}$ [see Eq. (4)]. If we assume high potential barriers between the second, the third, and the fourth atom, i.e., $J_{2}^{(a, b)}=J_{3}^{(a, b)}=0$, such that these atoms form a separate, decoupled system, we merely have to describe the dynamics of the first and the second atom. If we further assume that $U_{a, b}, U_{a b}$ are much larger than the hopping terms $J_{j}^{(a, b)}$ we can adiabatically eliminate all states with more than one atom in a lattice site. By choosing the parameters according to

$$
\begin{gathered}
J_{1}^{(a)}=J_{1}^{(b)}=J, \\
U_{a}=U_{b}=U_{a b}=U,
\end{gathered}
$$

we arrive at a the effective Hamiltonian

$$
H_{Z}=-\frac{J^{2}}{U}\left(\sigma_{1}^{x} \sigma_{2}^{x}+\sigma_{1}^{y} \sigma_{2}^{y}+\sigma_{1}^{z} \sigma_{2}^{z}+1_{12}\right)=-\frac{2 J^{2}}{U} V_{12}=\frac{2 J^{2}}{U} Z_{L}
$$

where we omitted a constant term. The time evolution of the system then generates the rotation

$$
R_{L}^{z}(\theta)=\exp \left(-\mathrm{i} \frac{\theta}{2} Z_{L}\right),
$$

where $\theta=4 J^{2} t / U \hbar$. Hence, the time for a $R_{L}^{z}(\pi)$ rotation is given by $t=\pi U \hbar / 4 J^{2}$. As an example we consider Na atoms in an optical lattice with a wavelength of $\lambda=514 \mathrm{~nm}$. The height of the transverse potential barriers are assumed to be $V_{y}=V_{z}=50 E_{\mathrm{R}}$, which is in the range of today's experiments [43]. Here, $E_{\mathrm{R}}=\hbar^{2}(2 \pi)^{2} / 2 m \lambda^{2}$ is the recoil energy and $m$ is the mass of the atoms. If we increase the $s$-wave scattering length by a factor of 5 using a Feshbach resonance and choose $V_{x}=7.7 E_{\mathrm{R}}$ between the first and second lattice site we get a ratio $U / J=75$ which is well in the regime where the adiabatic elimination is valid, as will be discussed in more detail below. Note that for these parameters the assumptions for the validity of the Bose-Hubbard Hamiltonian Eq. (15) are fulfilled. The hopping term is then calculated to be $J=0.033 E_{\mathrm{R}}$ which results in an operation time of $t=8.7 \mathrm{~ms}$ for the $R_{L}^{z}(\pi)$ rotation. We emphasize that we never leave the DFS during the rotation and it is expected that this time is much shorter than the decoherence time of the DFS qubit. 
Nevertheless, the time for a $R_{L}^{z}(\pi)$ rotation can be made considerably shorter by tuning the interaction between the register atoms to zero (e.g., via a Feshbach resonance). As we show in Appendix B, the qubits still remain in the DFS for all times. In this interaction-free case, the exact dynamics of the two atom system (i.e., without adiabatic elimination) results after a time $t=\pi \hbar / 2 J$ in the mapping

$$
|\alpha, \beta\rangle_{12} \rightarrow-|\beta, \alpha\rangle_{12}, \quad \alpha, \beta=0,1 .
$$

Up to a global phase this is equivalent to a $R_{L}^{z}(\pi)$ rotation on the logical qubits. The time needed for a lattice as above is $t=0.23 \mathrm{~ms}$. The disadvantage of this scheme is that only rotations with a phase of $\pi$ can be performed.

According to Eq. (3) the implementation of $H_{X}$ involves three atoms. In the same spirit as for the $Z_{L}$ gate we require that the potential barrier between the third and fourth lattice site is high, i.e., $J_{3}^{(a, b)}=0$. By choosing the remaining parameters as

$$
\begin{gathered}
J_{2}^{(a)}=\sqrt{2} J_{1}^{(a)}=\sqrt{2} J_{1}^{(b)}=J_{2}^{(b)} \equiv \sqrt{2} J, \\
U_{a}=U_{b}=U_{a b}=U,
\end{gathered}
$$

and requiring that $J / U \ll 1$, we again adiabatically eliminate all states with more than one atom in one well. A detailed calculation is given in Appendix A. The resulting Hamiltonian on the logical qubits is then given by

$$
H_{X}=-\frac{2 \sqrt{3} J^{2}}{U} X_{L}
$$

where we omitted a constant term. This Hamiltonian generates a rotation

$$
R_{L}^{x}(\theta)=\exp \left(-\mathrm{i} \frac{\theta}{2} X_{L}\right),
$$

where $\theta=-4 \sqrt{3} J^{2} t / U \hbar$. Taking the numbers of the above example, the time required for a $R_{L}^{x}(-\pi)$ rotation is $t=5.0 \mathrm{~ms}$.

\section{Numerical simulation}

To test the validity of the adiabatic elimination we simulated the system numerically. In case of $R_{L}^{z}$-rotations the parameters of the exact Hamiltonian Eq. (15) are chosen according to Eqs. (18) and (19). We numerically calculated the fidelity of the DFS qubit remaining in its state during an $R_{L}^{z}$-rotation,

$$
f_{Z} \equiv\left|\left\langle\left. l\right|_{L} \exp \left(-\mathrm{i} H_{Z} t / \hbar\right) \mid l\right\rangle_{L}\right|^{2}
$$

with $l=0,1$. For $U / J=75$ it turns out that the infidelity $1-f_{Z}$ is smaller than $3 \times 10^{-3}$ for $l=1$ and smaller than $10^{-9}$ for $l=0$ during the whole duration of a $R_{L}^{z}(2 \pi)$ rotation.

Since this fidelity does not take the phase difference $\phi$ between states $|0\rangle_{L}$ and $|1\rangle_{L}$ into account, $\phi$ was calculated as well. In Fig. 8 the phase difference is shown for the rotation $R_{L}^{z}(\pi)$. As expected, for increasing ratios $U / J$ the phase difference gets closer to $\pi$. For $U / J \approx 75$ the inaccuracy of the achieved phase is already smaller than $10^{-3}$, which shows that the adiabatic elimination is well justified.

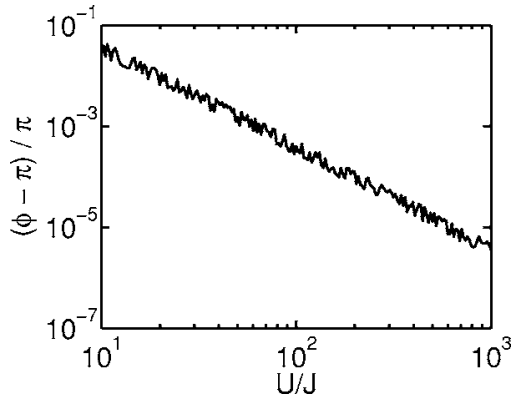

FIG. 8. Numerical studies of the phase difference $\phi$ between states $|0\rangle_{L}$ and $|1\rangle_{L}$ after applying the rotation $R_{L}^{z}(\pi)$ versus the ratio $U / J$ calculated with the full Hamiltonian Eq. (15) and the parameters Eqs. (18) and (19) given by adiabatic elimination.

From an experimental point of view it is also important to know what happens when some of the parameters are detuned. Some results are shown in Fig. 9. The error due to the nonvanishing hopping between the second and third lattice site $J_{2}$ is negligible as long as $J_{2}<10^{-2} J_{1}$. Because of this we have completely neglected the influence of the fourth register atom which is not involved in these rotations. In the case of a detuning of the interaction coefficient $U_{a b}$ the fidelity changes quite quickly if $U_{a b} / U$ differs from 1 , but is smaller than $10^{-3}$ as long as the detuning is less than $2 \%$.

The fidelity Eq. (27) and the phase difference $\phi$ between the logical qubit for the faster scheme of a $R_{L}^{z}(\pi)$ rotation for small, residual interactions $U / J$ (in the ideal case this interaction is vanishing) are shown in Fig. 10. For this case the inaccuracy of the gate decreases with decreasing $U / J$ and is smaller than $2 \times 10^{-3}$ for $U / J \approx 10^{-2}$. The error calculated according to Eq. (27) is orders of magnitudes smaller than the phase inaccuracy.

The simulations of the $R_{L}^{x}$ rotations lead to similar numbers. The fidelity of this process, determined by
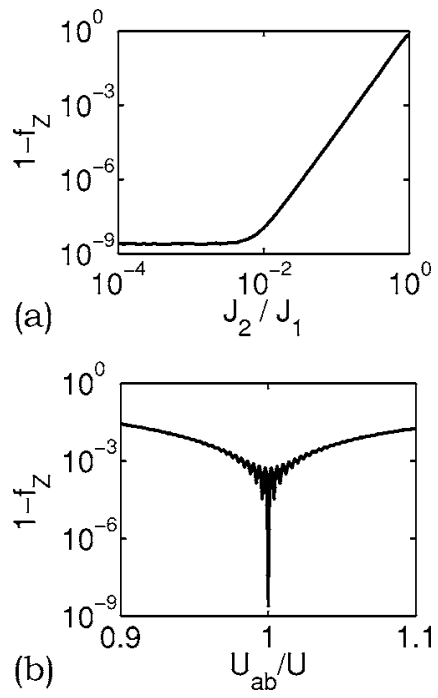

FIG. 9. Fidelity Eq. (27) for the rotation $R_{L}^{z}(\pi)$. In (a) the hopping term between the second and third atom is detuned, in (b) the interaction between register atoms of kind $a$ and $b$. The other parameters are (a) $U_{a, b}=U_{a b}=100 J_{1}$, (b) $U_{a, b}=U=100 \mathrm{~J}$. 


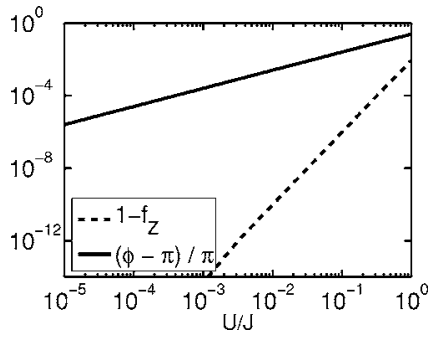

FIG. 10. Numerical studies for a fast rotation with no or only weak interaction between the atoms. The fidelity Eq. (27) of the rotation $R_{L}^{z}(\pi)$ and the phase difference between the two logical qubits after this rotation versus the ratio $U / J$ is calculated with the full Hamiltonian Eq. (15). The accuracy of the gate gets better with a smaller interaction $U$ between the atoms. Note that here no adiabatic elimination has been used.

$$
f_{X} \equiv\left|\left\langle\left. 0\right|_{L} \exp \left(-\mathrm{i} H_{X} t / \hbar\right) \mid 1\right\rangle_{L}\right|^{2}
$$

is shown in Fig. 11 depending on the ratio $U / J$. As can be seen from this figure, the infidelity $1-f_{X}$ decreases with increasing ratio of $U / J$ and for a $U / J \approx 75$ the infidelity is smaller than $10^{-3}$. Any infidelities because of a change of the relative phases between the two logical states are orders of magnitudes smaller and play no role.

Figure 12 shows the results of numerical simulations if various system parameters are detuned. For the case of a nonideal hopping the deviation should be smaller than $1.5 \%$ to yield infidelities smaller than $3 \times 10^{-3}$. A slight detuning in the interaction strength is less critical, but should nevertheless be smaller than $4 \%$ to achieve small errors.

\section{Controlled phase gate}

The CPHASE gate can be realized by a controlled swap operation between the third and the fourth atom since, as mentioned in Sec. II A $1, Z_{L}=-V_{34}$. We assume that the potential barriers between the first, second, and third atom of the DFS qubit are high enough to ignore the dynamics of the first two atoms. The auxiliary atom is assumed to be distinguishable from the DFS atoms and is confined in a sufficiently deep lattice such that it always remains at its site. If the auxiliary atom is moved to the free site of the DFS string (cf. Fig. 7) and if a register atom tunnels into the site of the

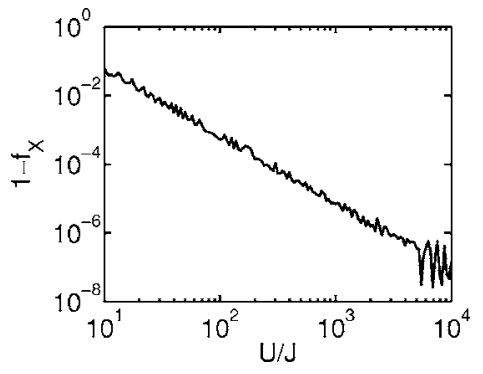

FIG. 11. Numerical studies of the fidelity Eq. (28) of the rotation $R_{L}^{x}(\pi)$ versus the ratio $U / J$ calculated with the full Hamiltonian Eq. (15) and the parameters Eqs. (23) and (24) given by adiabatic elimination.

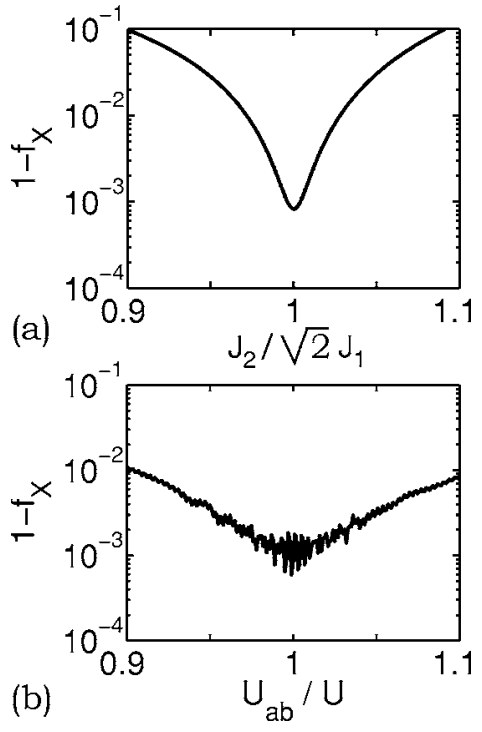

FIG. 12. Fidelity Eq. (28) for the rotation $R_{L}^{x}(\pi)$. In (a) the hopping term between the second and third atom is detuned, in (b) the interaction between register atoms of kind $a$ and $b$. The other parameters are (a) $U_{a, b}=U_{a b}=100 J_{1}$, (b) $U_{a, b}=U=100 \mathrm{~J}$.

auxiliary they interact with each other giving the Hamiltonian

$$
\hat{H}_{\mathrm{CPHASE}}=\hat{H}+\sum_{\sigma=0,1} U_{\sigma}^{q} \hat{a}_{4}^{\dagger} \hat{a}_{4} \hat{q}_{\sigma}^{\dagger} \hat{q}_{\sigma}+U_{\sigma}^{q} \hat{b}_{4}^{\dagger} \hat{b}_{4} \hat{q}_{\sigma}^{\dagger} \hat{q}_{\sigma}
$$

where $\hat{H}$ is the Hamiltonian given by Eq. (15), $\hat{q}_{\sigma}$ is the state-dependent annihilation operator of the auxiliary atom with $q$ its internal state, and $U_{\sigma}^{q}$ the interaction strength which is independent of the internal state of the DFS atoms. If the interaction $U_{0}^{q}$ for state $|0\rangle_{q}$ of the auxiliary atom is very large compared to the hopping strength, the third or fourth register atoms cannot enter the lattice site of the auxiliary atom and hence they will remain in their sites. Numerical tests show that during the gate operation this still holds for an interaction strength of $U_{0}^{q} / J \approx 100$, where $J$ is the hopping term of the register atoms. Under these circumstances the probability of finding the register in its original state is larger than $1-10^{-3}$. If the auxiliary atom is in the state $|1\rangle_{q}$ and $U_{1}^{q}=0$ the existence of the auxiliary atom can be ignored and we only need to solve the dynamics of two register atoms in three lattice sites. A similar scheme has been discussed in $[69,70]$, where an electromagnetically induced transparency-like configuration was used to control the interaction strength. We will investigate in the following the two limiting cases of very large and vanishing interaction strength between the register atoms.

\section{Large interaction between the register atoms}

For the case of large $U / J$ we calculate the fidelities of the swap operation,

$$
f_{01}=|\langle 0 \sigma 1|\mathcal{U}(t)| 1 \sigma 0\rangle|^{2}
$$




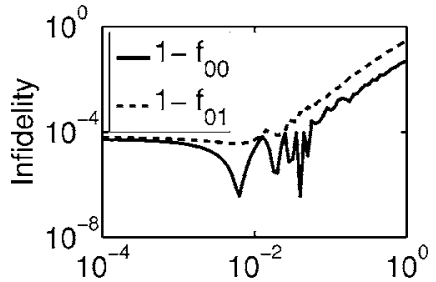

(a)

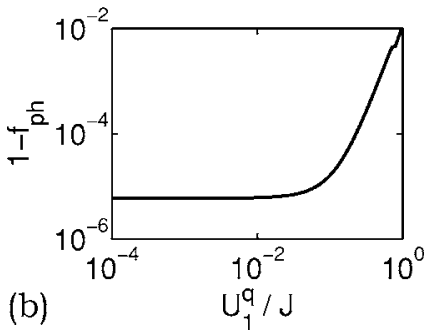

FIG. 13. (a) Numerical results for the fidelities Eqs. (30) and (31), where the auxiliary atom has a nonvanishing rest interaction with the register atoms. (b) Numerical results for the fidelity Eq. (35) due to the phase difference, where the auxiliary atom has a nonvanishing rest interaction with the register atoms. In both cases we took for the register atoms $U / J=100$.

$$
f_{00}=f_{11}=|\langle 0 \sigma 0|\mathcal{U}(t)| 0 \sigma 0\rangle|^{2}
$$

numerically, where $t$ is the numerically obtained time for the CPHASE gate and $\mathcal{U}(t)=\exp \left(-\mathrm{i} \hat{H}_{\mathrm{CPHASE}} t / \hbar\right)$ is the time evolution operator corresponding to Hamiltonian Eq. (29). The states $|\alpha \sigma \beta\rangle, \alpha, \beta=0,1$ denote the states of the third and the fourth DFS atom, $|\alpha\rangle$ and $|\beta\rangle$, and $|\sigma=1\rangle$ is the internal state of the auxiliary atom, located in between the two DFS atoms. We choose $J^{(a, b)}=J$ and $U_{a, b}=U_{a b}=U$.

In Fig. 13(a) the fidelities Eqs. (30) and (31) are shown for a small but nonvanishing interaction $U_{1}^{q}$ between the auxiliary atom and the register atoms for a ratio $U / J=100$. As can be seen the infidelities are smaller than $10^{-3}$ as long as the interaction fulfills $U_{1}^{q} / J<0.05$. A further numerical observation is that the value of $f_{00}\left(f_{01}\right)$ for very small $U_{1}^{q} / J$ gets smaller with increasing $U / J$.

The fidelities Eqs. (30) and (31) do not take into account the occurrence of phases. In general, the dynamics of the system will yield a phase,

$$
|\alpha \sigma \beta\rangle \rightarrow e^{\mathrm{i} \varphi_{\alpha \beta}}|\beta \sigma \alpha\rangle
$$

with $\alpha, \beta=0,1$. Since the interactions $U_{\sigma}^{q}$ do not depend on the state of the DFS atoms we have $\varphi_{01}=\varphi_{10}$ and $\varphi_{00}=\varphi_{11}$ and a nonvanishing $U_{1}^{q}$ leads to the behavior

$$
\mathcal{U}(t)|0\rangle_{L}=e^{i \varphi_{10}}|0\rangle_{L}
$$

$$
\mathcal{U}(t)|1\rangle_{L}=e^{i \varphi} \frac{1}{2 \sqrt{3}}\left[2(|1100\rangle+|0011\rangle)-e^{i \varphi}(|01\rangle+|10\rangle)^{\otimes 2}\right]
$$

of the four DFS atoms, i.e., to the presence of a nonzero phase $\varphi \equiv \varphi_{00}-\varphi_{10}$. Thus the state $\mathcal{U}(t)|1\rangle_{L}$ is not entirely
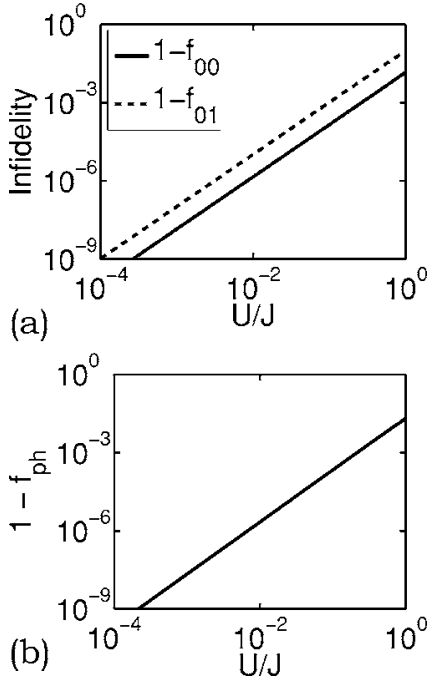

FIG. 14. (a) Fidelity after evolving the state for a time $t=\pi \hbar / \sqrt{2} J$ for the case of noninteracting register atoms. (b) Fidelity Eq. (35) of state $|1\rangle_{L}$ after the time evolution, where a perfect fidelity of the atom swap operation has been assumed and the error is only due to the phase difference, see text.

contained in the DFS anymore. To study this error we define the fidelity

$$
f_{\mathrm{ph}}=\left|\left\langle\left. 1\right|_{L} \mathcal{U}(t) \mid 1\right\rangle_{L}\right|^{2}=\frac{5+4 \cos \varphi}{9},
$$

which is shown in Fig. 13(b) depending on $U_{1}^{q} / J$. The infidelity $1-f_{\mathrm{ph}}$ is typically one order of magnitude smaller than the infidelities shown in Fig. 13(a).

The time which is needed to perform the swap gate has been calculated numerically. For a ratio of $U / J=75$ and the same lattice parameters as for the $R_{L}^{z}$ rotations we get $t=11.4 \mathrm{~ms}$.

Numerical tests show that for the same ratio $U / J=75$ the probability of finding the qubit outside the DFS is smaller than $5 \times 10^{-4}$ during the whole gate operation time.

\section{No interaction between DFS atoms}

A substantially shorter gate time can be achieved for the case of a vanishing interaction between the DFS atoms. In Appendix B it is shown that the qubits still remain in the DFS during the gate operation. By analytically solving the time evolution of the Hamiltonian it can be shown (see also Appendix B) that after a time $t=\pi \hbar / \sqrt{2} J$ the dynamics yields the mapping

$$
|\alpha \sigma \beta\rangle \rightarrow|\beta \sigma \alpha\rangle, \quad \alpha, \beta=0,1, \quad \sigma=1
$$

which is a swap operation between the third and the fourth DFS atom and thus a CPHASE gate. For the same lattice potential as earlier with $V_{x}=7.7 E_{\mathrm{R}}$ the gate time is given by $t=0.33 \mathrm{~ms}$.

The results of numerical tests for a small rest interaction between the register atoms are shown in Fig. 14. Figure 14(a) shows the infidelities derived form Eqs. (30) and (31) and Fig. 14(b) shows the infidelity derived from Eq. (35) all 
depending on $U / J$. The fidelities are better than $1-10^{-3}$ for interactions $U / J<0.05$. The results of numerical simulations for a nonvanishing rest interaction between the DFS atoms and a state $|1\rangle_{q}$ of the auxiliary atom yield an infidelity smaller that $3 \times 10^{-3}$ for $U_{1}^{q} / J \leqslant 0.05$.

In conclusion, a reliable CPHASE gate can be implemented in both cases. If the auxiliary atom is in the state $|1\rangle_{q}$ and thus a swap of register atoms 3 and 4 should occur, the interaction $U_{1}^{q}$ between the auxiliary atom and the register atoms is required to be smaller than $0.05 \mathrm{~J}$. If the auxiliary atom is in the state $|0\rangle_{q}$ no swap should occur. This holds during the gate interaction time for an interaction $U_{0}^{q}$ larger than approximately $100 \mathrm{~J}$. The difference of more than three orders of magnitude in the interaction can be achieved either by using a Feshbach resonance or by using an electromagnetically induced transparency scheme as in [69].

\section{Gate times and decoherence}

Recent experiments have demonstrated that the decoherence time of quantum information stored in DFSs can be increased by several orders of magnitudes compared to unprotected qubits. In the case of ion traps decoherence times of more than $7 \mathrm{~s}$ have been reported [10] and although the DFS scheme in this experiment was different from ours we expect a similar improvement in the reliability of our quantum memory. The required storage time is found by adding the single qubit and CPHASE gate times calculated in previous sections. According to these the state transfer from the auxiliary atom to the DFS takes $11 \mathrm{~ms}$, neglecting the time which is needed to implement the rotation and measurement of the auxiliary atom. The entanglement purification module described in Fig. 4 takes 26 ms, the CNOT operation of Fig. 5 takes $28 \mathrm{~ms}$. The necessary readout process of the DFS qubits takes $11 \mathrm{~ms}$. In comparison to these times the additional operations in steps (i), (ii), and (iii) of Fig. 1 are expected to contribute a negligible amount of extra time to the overall scheme $[31,32,58-62]$. All these times are very short compared to the expected decoherence time of the DFS qubit. Since the system remains in the DFS during the entire single qubit operations we conclude that decoherence of the DFS qubits plays only a minor role.

However, in the modules which involve a CPHASE gate between the auxiliary qubit and a DFS qubit the decoherence of the auxiliary atom has to be taken into account since, in general, it gets entangled with the DFS qubit thus lowering the overall fidelity. As discussed in Sec. II B the fidelity for, e.g., the CNOT gate is better than $1-\gamma t$, where $\gamma$ is the inverse decoherence time and $t$ is the time to perform a CPHASE gate. In our fastest example (without interaction between register atoms) a CPHASE gate takes approximately $0.33 \mathrm{~ms}$. In Ref. [63] decoherence times of $146 \mathrm{~ms}$ for $\sim 50 \mathrm{Cs}$ atoms stored in a red-detuned standing wave have been reported. In the experiment presented in Ref. [52] the influence of a moving conveyor belt is investigated. It is found that the transport procedure decreases the coherence time by approximately a factor of 2 . This would still be long enough for our purposes. For the entanglement purification module, which has the worst fidelity of all our models, the influence of the auxiliary atom leads to a fidelity of better than $98.7 \%$. For the other modules the fidelity due to decoherence of the auxiliary atom is better than $99 \%$. The usage of a blue-detuned trap could give further considerable increase of the decoherence time. Aside from this, it should be emphasized that all these times have been measured for many atoms in a trap. In the case of single atoms longer decoherence times can be expected. Thus, the influence of the auxiliary atom on the modules described in Sec. II A is small and only slightly decreases their fidelity.

\section{CONCLUSION}

In this paper we developed a scheme for the robust implementation of a quantum repeater. The qubits at the repeater nodes are encoded in a DFS which increases the decoherence time of the quantum memory considerably and thus improves the reliability of the scheme. We showed that the quantum repeater can be divided into simple modules whose implementation only requires a small number of different quantum gate operations. The single qubit gates can be realized by selectively lowering potential barriers between the atoms without ever leaving the DFS. Controlled operations are mediated by a single auxiliary qubit which is not decoherence free. However, the influence of this qubit on the gate fidelities was thoroughly investigated and shown to be sufficiently small as not to significantly affect the quality of the repeater scheme.

Furthermore, we presented proposals for implementing the DFS qubits and all necessary operations in systems of neutral atoms trapped in arrays of optical dipole traps. The times for implementing these gates were calculated and shown to be much smaller than the expected decoherence time of the DFS qubits. Numerical examinations have shown that the achieved gate fidelities can be better than $98.7 \%$ and that the gate operations are stable against possible, small deviations of the system parameters.

Our scheme is extendable to general purpose quantum computing with DFS qubits, since the elementary one-qubit rotations $R_{L}^{x}, R_{L}^{z}$ and a two-qubit gate, which together form a universal set of gates, can be realized. However, during the CPHASE gate the DFS is left. This effect is very small for our quantum repeater scheme and the influence of leaving the DFS in other applications as well as the scalability of the scheme is the subject of further investigations.

Recent experiments have demonstrated that it is possible to implement and control quantum registers with a few atoms in optical lattices [71] or dipole trap arrays [45] and that the decoherence times of the auxiliary atom are long enough to ensure a successful operation of our scheme [52,63]. The experimental combination of atom registers and optical conveyor belts is also planned [71]. Hence we are confident that the presented scheme can be implemented with current and near future experimental techniques.

\section{ACKNOWLEDGMENTS}

A.K. thanks Stephen R. Clark for useful discussions and acknowledges financial support from the Keble Association. 
This work was supported by the EPSRC (UK) through the QIP IRC (GR/S82176/01) and project EP/C51933/1. The research was also supported by the EU through the STREP project OLAQUI. The research of U.D. was supported by a Marie Curie Intra-European grant (EIF) within the 6th European Community Framework Programme ('RAQUIN'). The contents of this paper reflect the author's views and not the views of the European Commission. C.M.A. was supported by the Fundação para a Ciência e Tecnologia (Portugal).

\section{APPENDIX A: ADIABATIC ELIMINATION FOR THE $X_{L}$ GATE}

The derivation of Hamiltonian $H_{X}$, Eq. (25), is given in more detail. The starting point is the Hamiltonian Eq. (15) for three atoms in three lattice sites. Since the number of atoms in state $a$ and $b$ is conserved the states decouple in two subspaces where the first one contains only states with one atom in state $a$ and two atoms in state $b$ and the other one contains two atoms in state $a$ and one atom in state $b$. The states with three atoms in the same internal state do not occur in our system. For simplicity we only consider the second subset, the results for the other subset can be derived in the same way.

Let $Q$ be the projection operator on all states with more than one atom in a lattice site, $P=1-Q$. The Hamiltonian after adiabatic elimination in the first order perturbation theory is given by

$$
\hat{H}_{\text {eff }}=\lim _{\varepsilon \rightarrow 0} P \hat{H} P-P \hat{H} Q(Q \hat{H} Q+\varepsilon 1)^{-1} Q \hat{H} P .
$$

The inversion of $Q \hat{H} Q+\varepsilon \perp$ was calculated up to first order in the perturbation parameter $J / U$ since in this treatment higher orders of $J / U$ are neglected anyway. In the basis $\{|100\rangle,|010\rangle,|001\rangle\}$ the adiabatically eliminated Hamiltonian has the form

$$
\hat{H}_{\text {eff }}=\left(\begin{array}{ccc}
f_{1} & g_{1} & 0 \\
g_{1} & f_{2} & g_{2} \\
0 & g_{2} & f_{3}
\end{array}\right),
$$

with

$$
\begin{gathered}
f_{1}=-\frac{U_{a}\left[\left(J_{1}^{(a)}\right)^{2}+\left(J_{1}^{(b)}\right)^{2}\right]+4 U_{a b}\left(J_{2}^{(a)}\right)^{2}}{U_{a} U_{a b}}, \\
f_{2}=-\frac{\left(J_{1}^{(a)}\right)^{2}+\left(J_{2}^{(a)}\right)^{2}+\left(J_{1}^{(b)}\right)^{2}+\left(J_{2}^{(b)}\right)^{2}}{U_{a b}}, \\
f_{3}=-\frac{U_{a}\left[\left(J_{2}^{(a)}\right)^{2}+\left(J_{2}^{(b)}\right)^{2}\right]+4 U_{a b}\left(J_{1}^{(a)}\right)^{2}}{U_{a} U_{a b}}, \\
g_{1}=-2 \frac{J_{1}^{(a)} J_{1}^{(b)}}{U_{a b}},
\end{gathered}
$$

$$
g_{2}=-2 \frac{J_{2}^{(a)} J_{2}^{(b)}}{U_{a b}} .
$$

The action of this Hamiltonian on the logical qubits can be written in the form

$$
\begin{gathered}
\hat{H}_{\mathrm{eff}}|0\rangle_{L}=u|0\rangle_{L}+v|1\rangle_{L}, \\
\hat{H}_{\mathrm{eff}}|1\rangle_{L}=u|1\rangle_{L}+v|0\rangle_{L} .
\end{gathered}
$$

Solving the corresponding equations for $u$ and $v$ one finds

$$
\begin{gathered}
u=f_{2}, \\
v=\frac{\sqrt{3}}{2} g_{2}, \\
f_{1}=f_{2}+2 g_{1}, \\
g_{2}=2 g_{1}, \\
f_{3}=f_{2}+g_{1} .
\end{gathered}
$$

Because of the symmetry between the states with two atoms in state $a$ and one in state $b$ and vice versa the only physical solution for these equations is given by Eqs. (23)-(25).

\section{APPENDIX B: QUBITS REMAIN IN THE DFS}

In this appendix we show that the DFS qubits remain decoherence free in the case without any interaction between the physical qubits, which is relevant for the fast $R_{L}^{z}(\pi)$ rotation and the CPHASE gate. Since in this case there can be more than one atom in a lattice site we introduce Fock states $\left|n_{a}^{1}, n_{b}^{1} ; n_{a}^{2}, n_{b}^{2} ; \ldots ; n_{a}^{5}, n_{b}^{5}\right\rangle$, where $n_{a}^{j}\left(n_{b}^{j}\right)$ is the number of atoms in mode $a(b)$ in lattice site $j=1, \ldots, 5$, respectively.

The fast $R_{L}^{z}(\pi)$ rotation can be performed by setting $U_{a, b}=U_{a b}=0, J_{2}^{(a, b)}=J_{3}^{(a, b)}=J_{4}^{(a, b)}=0$. In this case the corresponding time evolution operator $U(t)=\exp (-\mathrm{i} \hat{H} t / \hbar)$, where $\hat{H}$ is given by Eq. (15), can be calculated analytically and we get

$$
U(t)|0\rangle_{L}=|0\rangle_{L},
$$

for all $t$ and thus the system never leaves the DFS. The state $|1\rangle_{L}$ evolves as

$$
\begin{aligned}
U(t)|1\rangle_{L}= & \cos \left(\frac{2 J t}{\hbar}\right)|1\rangle_{L}+\frac{\mathrm{i}}{2 \sqrt{3}} \sin \left(\frac{2 J t}{\hbar}\right)\left\{\frac{2}{\sqrt{2}}[|0,2 ; 0,0\rangle\right. \\
& +|0,0 ; 0,2\rangle] \otimes|1,0 ; 0,0 ; 1,0\rangle+\frac{2}{\sqrt{2}}[|2,0 ; 0,0\rangle \\
& +|0,0 ; 2,0\rangle] \otimes|0,1 ; 0,0 ; 0,1\rangle-[|1,1 ; 0,0\rangle \\
& +|0,0 ; 1,1\rangle] \otimes[|1,0 ; 0,0 ; 0,1\rangle+|0,1 ; 0,0 ; 1,0\rangle]\} .
\end{aligned}
$$


Clearly, the first term $\cos (2 J t / \hbar)|1\rangle_{L}$ remains in the DFS. In order to see that this is also true for the second part we rewrite the states in first quantization. Let $|\alpha \beta\rangle_{A}$ denote a state where the first atom is in state $|\alpha\rangle$ and the second one in state $|\beta\rangle$, independent in which lattice site they are stored. Due to symmetrization we get

$$
\frac{1}{\sqrt{2}}[|1,1 ; 0,0\rangle+|0,0 ; 1,1\rangle] \rightarrow \frac{1}{\sqrt{2}}\left[|01\rangle_{A}+|10\rangle_{A}\right],
$$

$$
\frac{1}{\sqrt{2}}[|2,0 ; 0,0\rangle+|0,0 ; 2,0\rangle] \rightarrow|00\rangle_{A},
$$

$$
\frac{1}{\sqrt{2}}[|0,2 ; 0,0\rangle+|0,0 ; 0,2\rangle] \rightarrow|11\rangle_{A} .
$$

By rewriting Eq. (B2) we get

$$
\begin{aligned}
U(t)|1\rangle_{L}= & \cos \left(\frac{2 J t}{\hbar}\right)|1\rangle_{L}+\frac{\mathrm{i}}{2 \sqrt{3}} \sin \left(\frac{2 J t}{\hbar}\right)\left\{2|11\rangle_{A} \otimes|00\rangle_{A}\right. \\
& +2|00\rangle_{A} \otimes|11\rangle_{A}-\left[|01\rangle_{A}+|10\rangle_{A}\right] \otimes\left[|01\rangle_{A}\right. \\
& \left.\left.+|10\rangle_{A}\right]\right\} .
\end{aligned}
$$

The part proportional to the sine function is again decoherence free, because it is just the state $|1\rangle_{L}$.

The case of the CPHASE gate is more complicated because the atoms can hop into an additional lattice site. The time evolution of state $|0\rangle_{L}$ is given by

$$
\begin{aligned}
U(t)|0\rangle_{L}= & {[|1,0 ; 0,1\rangle-|0,1 ; 1,0\rangle] \otimes\left\{\cos \left(\frac{\sqrt{2} J t}{\hbar}\right)[|1,0 ; 0,0 ; 0,1\rangle-|0,1 ; 0,0 ; 1,0\rangle]+\frac{\mathrm{i}}{\sqrt{2}} \sin \left(\frac{\sqrt{2} J t}{\hbar}\right)[|1,0 ; 0,1 ; 0,0\rangle\right.} \\
& -|0,1 ; 1,0 ; 0,0\rangle+|0,0 ; 1,0 ; 0,1\rangle-|0,0 ; 0,1 ; 1,0\rangle]\} .
\end{aligned}
$$

For $|1\rangle_{L}$ we get

$$
\begin{aligned}
U(t)|1\rangle_{L}= & {[|1,0 ; 1,0\rangle+|0,1 ; 0,1\rangle] \otimes\left\{\frac{1}{4}\left[3+\cos \left(\frac{2 \sqrt{2} J t}{\hbar}\right)\right][|0,1 ; 0,0 ; 1,0\rangle+|1,0 ; 0,0 ; 0,1\rangle]\right.} \\
& +\frac{\mathrm{i}}{2 \sqrt{2}} \sin \left(\frac{2 \sqrt{2} J t}{\hbar}\right)[|0,0 ; 0,1 ; 1,0\rangle+|0,0 ; 1,0 ; 0,1\rangle+|0,1 ; 1,0 ; 0,0\rangle+|1,0 ; 0,1 ; 0,0\rangle] \\
& \left.-\frac{1}{2} \sin ^{2}\left(\frac{\sqrt{2} J t}{\hbar}\right)[|1,1 ; 0,0 ; 0,0\rangle+2|0,0 ; 1,1 ; 0,0\rangle+|0,0 ; 0,0 ; 1,1\rangle]\right\} \\
& +|0,1 ; 0,1\rangle \otimes\left\{\frac{1}{4}\left[3+\cos \left(\frac{2 \sqrt{2} J t}{\hbar}\right)\right]|1,0 ; 0,0 ; 1,0\rangle+\frac{\mathrm{i}}{2 \sqrt{2}} \sin \left(\frac{2 \sqrt{2} J t}{\hbar}\right)[|1,0 ; 1,0 ; 0,0\rangle+|0,0 ; 1,0 ; 1,0\rangle]\right. \\
& \left.-\frac{1}{2 \sqrt{2}} \sin ^{2}\left(\frac{\sqrt{2} J t}{\hbar}\right)[|2,0 ; 0,0 ; 0,0\rangle+2|0,0 ; 2,0 ; 0,0\rangle+|0,0 ; 0,0 ; 2,0\rangle]\right\} \\
& +|1,0 ; 1,0\rangle \otimes\left\{\frac{1}{4}\left[3+\cos \left(\frac{2 \sqrt{2} J t}{\hbar}\right)\right]|0,1 ; 0,0 ; 0,1\rangle+\frac{\mathrm{i}}{2 \sqrt{2}} \sin \left(\frac{2 \sqrt{2} J t}{\hbar}\right)[|0,1 ; 0,1 ; 0,0\rangle+|0,0 ; 0,1 ; 0,1\rangle]\right. \\
& \left.-\frac{1}{2 \sqrt{2}} \sin ^{2}\left(\frac{\sqrt{2} J t}{\hbar}\right)[|0,2 ; 0,0 ; 0,0\rangle+2|0,0 ; 0,2 ; 0,0\rangle+|0,0 ; 0,0 ; 0,2\rangle]\right\}
\end{aligned}
$$

Making analogous substitutions as above we see that these qubits are again decoherence-free at all times.

[1] P. W. Shor, Phys. Rev. A 52, R2493 (1995).

[2] A. M. Steane, Phys. Rev. Lett. 77, 793 (1996).

[3] A. Ekert and C. Macchiavello, Phys. Rev. Lett. 77, 2585 (1996).

[4] E. Knill, R. Laflamme, and W. H. Zurek, Science 279, 342 (1998).
[5] P. Zanardi and M. Rasetti, Phys. Rev. Lett. 79, 3306 (1997).

[6] L.-M. Duan and G.-C. Guo, Phys. Rev. A 57, 737 (1998).

[7] D. A. Lidar, I. L. Chuang, and K. B. Whaley, Phys. Rev. Lett. 81, 2594 (1998).

[8] D. Bacon, J. Kempe, D. A. Lidar, and K. B. Whaley, Phys. Rev. Lett. 85, 1758 (2000). 
[9] D. Kielpinski, V. Meyer, M. A. Rowe, C. A. Sackett, W. M. Itano, C. Monroe, and D. J. Wineland, Science 291, 1013 (2001).

[10] C. Langer, R. Ozeri, J. D. Jost, J. Chiaverini, B. DeMarco, A. Ben-Kish, R. B. Blakestad, J. Britton, D. B. Hume, W. M. Itano, D. Leibfried, R. Reichle, T. Rosenband, T. Schaetz, P. O. Schmidt, and D. J. Wineland, Phys. Rev. Lett. 95, 060502 (2005).

[11] E. M. Fortunato, L. Viola, J. Hodges, G. Teklemariam, and D. G. Cory, New J. Phys. 4, 5 (2002).

[12] J. E. Ollerenshaw, D. A. Lidar, and L. E. Kay, Phys. Rev. Lett. 91, 217904 (2003).

[13] L. Viola, E. M. Fortunato, M. A. Pravia, E. Knill, R. Laflamme, and D. G. Cory, Science 293, 2059 (2001).

[14] P. G. Kwiat, A. J. Berglund, J. B. Altepeter, and A. G. White, Science 290, 498 (2000).

[15] M. Mohseni, J. S. Lundeen, K. J. Resch, and A. M. Steinberg, Phys. Rev. Lett. 91, 187903 (2003).

[16] M. Bourennane, M. Eibl, S. Gaertner, C. Kurtsiefer, A. Cabello, and H. Weinfurter, Phys. Rev. Lett. 92, 107901 (2004).

[17] H.-J. Briegel, W. Dür, J. I. Cirac, and P. Zoller, Phys. Rev. Lett. 81, 5932 (1998).

[18] L.-M. Duan, M. D. Lukin, J. I. Cirac, and P. Zoller, Nature (London) 414, 413 (2001).

[19] J. K. Pachos and P. L. Knight, Phys. Rev. Lett. 91, 107902 (2003).

[20] T. Calarco, E. A. Hinds, D. Jaksch, J. Schmiedmayer, J. I. Cirac, and P. Zoller, Phys. Rev. A 61, 022304 (2000).

[21] T. Calarco, U. Dorner, P. Julienne, C. Williams, and P. Zoller, Phys. Rev. A 70, 012306 (2004).

[22] A. K. Ekert, Phys. Rev. Lett. 67, 661 (1991).

[23] C. H. Bennett, G. Brassard, C. Crépeau, R. Jozsa, A. Peres, and W. K. Wootters, Phys. Rev. Lett. 70, 1895 (1993).

[24] M. Zukowski, A. Zeilinger, M. A. Horne, and A. K. Ekert, Phys. Rev. Lett. 71, 4287 (1993).

[25] C. H. Bennett, G. Brassard, S. Popescu, B. Schumacher, J. A. Smolin, and W. K. Wootters, Phys. Rev. Lett. 76, 722 (1996).

[26] D. Deutsch, A. Ekert, R. Jozsa, C. Macchiavello, S. Popescu, and A. Sanpera, Phys. Rev. Lett. 77, 2818 (1996).

[27] J.-W. Pan, S. Gasparoni, R. Ursin, G. Weihs, and A. Zeilinger, Nature (London) 423, 417 (2003).

[28] T. Yamamoto, M. Koashi, S. K. Özdemir, and N. Imoto, Nature (London) 421, 343 (2003).

[29] P. G. Kwiat, S. Barraza-Lopez, A. Stefanov, and N. Gisin, Nature (London) 409, 1014 (2001).

[30] Z. Zhao, T. Yang, Y.-A. Chen, A.-N. Zhang, and J.-W. Pan, Phys. Rev. Lett. 90, 207901 (2003).

[31] J. I. Cirac, P. Zoller, H. J. Kimble, and H. Mabuchi, Phys. Rev. Lett. 78, 3221 (1997).

[32] S. J. van Enk, J. I. Cirac, and P. Zoller, Phys. Rev. Lett. 78, 4293 (1997).

[33] M. Fleischhauer and M. D. Lukin, Phys. Rev. Lett. 84, 5094 (2000)

[34] D. F. Phillips, A. Fleischhauer, A. Mair, R. L. Walsworth, and M. D. Lukin, Phys. Rev. Lett. 86, 783 (2001).

[35] D. N. Matsukevich, T. Chaneliere, S. D. Jenkins, S.-Y. Lan, T. Kennedy, and A. Kuzmich (2005), e-print quant-ph/0511012.

[36] W. Dür, H.-J. Briegel, J. I. Cirac, and P. Zoller, Phys. Rev. A 59, 169 (1999).

[37] C. H. Bennett, D. P. DiVincenzo, J. A. Smolin, and W. K.
Wootters, Phys. Rev. A 54, 3824 (1996).

[38] D. Jaksch, C. Bruder, J. I. Cirac, C. W. Gardiner, and P. Zoller, Phys. Rev. Lett. 81, 3108 (1998).

[39] For a review see D. Jaksch and P. Zoller, Ann. Phys. (N.Y.) 315, 52 (2005).

[40] J. I. Cirac and P. Zoller, Phys. Today 57(3), 38 (2004).

[41] L. Santos, M. A. Baranov, J. I. Cirac, H. U. Everts, H. Fehrmann, and M. Lewenstein, Phys. Rev. Lett. 93, 030601 (2004).

[42] B. Darquié, M. P. A. Jones, J. Dingjan, J. Beugnon, S. Bergamini, Y. Sortais, G. Messin, A. Browaeys, and P. Grangier, Science 454, 309 (2005).

[43] S. Fölling, F. Gerbier, A. Widera, O. Mandel, T. Gericke, and I. Bloch, Nature (London) 434, 481 (2005).

[44] R. Dumke, T. Müther, M. Volk, W. Ertmer, and G. Birkl, Phys. Rev. Lett. 89, 220402 (2002).

[45] R. Dumke, M. Volk, T. Müther, F. B. J. Buchkremer, G. Birkl, and W. Ertmer, Phys. Rev. Lett. 89, 097903 (2002).

[46] M. Greiner, O. Mandel, T. Esslinger, T. W. Hänsch, and I. Bloch, Nature (London) 415, 39 (2002).

[47] T. Stöferle, H. Moritz, C. Schori, M. Köhl, and T. Esslinger, Phys. Rev. Lett. 92, 130403 (2004).

[48] A. Widera, O. Mandel, M. Greiner, S. Kreim, T. W. Hänsch, and I. Bloch, Phys. Rev. Lett. 92, 160406 (2004).

[49] O. Mandel, M. Greiner, A. Widera, T. Tom, T. W. Hänsch, and I. Bloch, Nature (London) 425, 937 (2003).

[50] S. Nußmann, M. Hijlkema, B. Weber, F. Rohde, G. Rempe, and A. Kuhn, Phys. Rev. Lett. 95, 173602 (2005).

[51] J. A. Sauer, K. M. Fortier, M. S. Chang, C. D. Hamley, and M. S. Chapman, Phys. Rev. A 69, 051804(R) (2004).

[52] S. Kuhr, W. Alt, D. Schrader, I. Dotsenko, Y. Miroshnychenko, W. Rosenfeld, M. Khudaverdyan, V. Gomer, A. Rauschenbeutel, and D. Meschede, Phys. Rev. Lett. 91, 213002 (2003).

[53] S. Kuhr, W. Alt, D. Schrader, M. Müller, V. Gomer, and D. Meschede, Science 293, 278 (2001).

[54] D. Jaksch, H.-J. Briegel, J. I. Cirac, C. W. Gardiner, and P. Zoller, Phys. Rev. Lett. 82, 1975 (1999).

[55] D. G. Grier, Nature (London) 424, 810 (2003).

[56] S. Bergamini, B. Darquié, M. Jones, L. Jacubowiez, A. Browaeys, and P. Grangier, J. Opt. Soc. Am. B 21, 1889 (2004)

[57] U. Dorner, T. Calarco, P. Zoller, A. Browaeys, and P. Grangier, J. Opt. B: Quantum Semiclassical Opt. 7, S341 (2005).

[58] J.-W. Pan, D. Bouwmeester, H. Weinfurter, and A. Zeilinger, Phys. Rev. Lett. 80, 3891 (1998).

[59] T. Jennewein, G. Weihs, J.-W. Pan, and A. Zeilinger, Phys. Rev. Lett. 88, 017903 (2002).

[60] F. Sciarrino, E. Lombardi, G. Milani, and F. De Martini, Phys. Rev. A 66, 024309 (2002).

[61] N. Takei, H. Yonezawa, T. Aoki, and A. Furusawa, Phys. Rev. Lett. 94, 220502 (2005).

[62] H. de Riedmatten, I. Marcikic, J. A. W. van Houwelingen, W. Tittel, H. Zbinden, and N. Gisin, Phys. Rev. A 71, 050302(R) (2005).

[63] S. Kuhr, W. Alt, D. Schrader, I. Dotsenko, Y. Miroshnychenko, A. Rauschenbeutel, and D. Meschede, Phys. Rev. A 72, 023406 (2005).

[64] J. Kempe, D. Bacon, D. A. Lidar, and K. B. Whaley, Phys. Rev. A 63, 042307 (2001).

[65] M. A. Nielsen and I. L. Chuang, Quantum Computation and 
Quantum Information (Cambridge University Press, Cambridge, 2000).

[66] G. Palma, K. Suominen, and A. Ekert, Proc. R. Soc. London, Ser. A 452, 567 (1996).

[67] L.-M. Duan, E. Demler, and M. D. Lukin, Phys. Rev. Lett. 91, 090402 (2003).

[68] U. Dorner, P. Fedichev, D. Jaksch, M. Lewenstein, and P. Zoller, Phys. Rev. Lett. 91, 073601 (2003).
[69] A. Micheli, A. J. Daley, D. Jaksch, and P. Zoller, Phys. Rev. Lett. 93, 140408 (2004).

[70] A. J. Daley, S. R. Clark, D. Jaksch, and P. Zoller, Phys. Rev. A 72, 043618 (2005).

[71] D. Schrader, I. Dotsenko, M. Khudaverdyan, Y. Miroshnychenko, A. Rauschenbeutel, and D. Meschede, Phys. Rev. Lett. 93, 150501 (2004). 